\title{
Some Provably Hard Crossing Number Problems*
}

\author{
Daniel Bienstock \\ Department of IEOR, Columbia University, New York, NY 10027, USA
}

\begin{abstract}
This paper presents a connection between the problem of drawing a graph with the minimum number of edge crossings, and the theory of arrangements of pseudolines, a topic well-studied by combinatorialists. In particular, we show that any given arrangement can be forced to occur in every minimum crossing drawing of an appropriate graph. Using some recent results of Goodman, Pollack, and Sturmfels, this yields that there exists no polynomial-time algorithm for producing a straight-line drawing of a graph, which achieves the minimum number of crossings from among all such drawings. While this result has no bearing on the $P$ versus NP question, it is fairly negative with regard to applications. We also study the problem of drawing a graph with polygonal edges, to achieve the (unrestricted) minimum number of crossings. Here we obtain a tight bound on the smallest number of breakpoints which are required in the polygonal lines.
\end{abstract}

\section{Introduction}

A drawing of a simple graph $G$ is a subset of the plane $\mathbb{R}^{2}$ where each vertex is represented by a different point, and each edge is represented by a homeomorph of the closed unit interval $I^{1}$ with appropriate ends. Further, the drawings of any two edges meet at most once, and if they do, then either the two edges are incident to a common vertex, where their drawings meet, or the two drawings cross at their intersection point (the term cross is assumed to be understood). The crossing number problem consists of producing a drawing of $G$ which achieves the least possible number of crossings (this parameter is called the crossing number of $G$ and is denoted by $\operatorname{cr}(G)$ ). This problem is of interest in VLSI theory and in wiring layout problems (see [17]), and it has long been of interest in the graph theory

\footnotetext{
* This work was partially supported by the Center for Telecommunications Research, Columbia University.
} 
community (see [4], [22], and [23]). Computing the crossing number was shown to be NP-hard by Garey and Johnson [5] (the decision problem is in NP).

In many of the applications, it is further desirable that the edges be drawn as straight-line segments, with no restriction to orientation. Such a drawing has classically been called rectilinear, and the minimum number of crossings in a rectilinear drawing is called the rectilinear crossing number, denoted $\mathrm{cr}_{1}(G)$. We remark that practically every paper on crossing numbers has in fact also dealt with rectilinear crossing numbers (the latter being used to study the former). Using the proof in [5], it can be shown that computing the rectilinear crossing number is NP-hard. While this problem is not yet known to be in NP, it is clear that the (cartesian) coordinates of the vertices in a drawing can be assumed to be rational and, thus, integral. A generalization of the rectilinear crossing number is the $t$-polygonal crossing number (where $t \geq 1$ is an integer), denoted $\operatorname{cr}_{1}(G)$, which arises when every edge must be drawn as a $t$-polygonal line, i.e., a polygonal line with at most $t$ segments. Thus $t=1$ yields the rectilinear case. We see below the motivation for studying $t$-polygonal drawings of graphs with $t>1$.

In this paper we study the connection between crossing number problems and the theory of arrangements of pseudolines, an area of high interest in combinatorial geometry, which has most notably been studied by Grünbaum, Goodman, Pollack, and others (see [6]-[15]). For the purposes of this paper, a pseudoline is a homeomorph in $\mathbb{R}^{2}$ of the closed unit interval $I^{1}$. An arrangement $A$ of pseudolines is a collection of pseudolines, every two meeting at exactly one interior point, where they cross, and such that the ends of each pseudoline are in the closure of the unbounded component of $\mathbb{R}^{2} \backslash A$ (with the obvious abuse of notation here). Further, all arrangements considered here are simple, meaning that no three of the pseudolines meet at a common point. Following Grünbaum [15] we notice that an arrangement defines a two-dimensional cell complex (the unbounded face can be disregarded here). Two arrangements are said to be isomorphic if the labeled cell complexes they define are isomorphic (in which case we say one realizes the other, or is a realization of the other). A t-polygonal realization of an arrangement is one where each pseudoline is $t$-polygonal. Thus, a 1-polygonal realization is a straight-line realization.

We denote the vertex set of a graph $G$ by $V(G)$, and its edge set by $E(G)$. A plane graph is a planar graph, given in a particular embedding in the plane.

Remark. The same planar graph can yield different plane graphs.

Our first result is as follows (stated in abridged form here):

Theorem 1. Let $A$ be an arrangement of $n$ pseudolines. There is a graph $G_{A}$, so that:

(i) Every drawing of $G_{A}$ with $\operatorname{cr}\left(G_{A}\right)$ crossings contains a realization of $A$. Further, $\operatorname{cr}\left(G_{A}\right)=5 n(n-1)$ and $\left|E\left(G_{A}\right)\right|=O\left(n^{3}\right)$.

(ii) Let $t \geq 1$. If $A$ has a t-polygonal realization, then every t-polygonal drawing of $G_{A}$ with $\mathrm{cr}_{t}\left(G_{A}\right)$ crossings contains a $t$-polygonal realization of $A$. Further, $\operatorname{cr}_{t}\left(G_{A}\right)=5 n(n-1)$ and $\left|E\left(G_{A}\right)\right|=O\left(t n^{4}\right)\left(O\left(n^{3}\right)\right.$ for $\left.t=1\right)$. 
Thus if we can construct arrangements all of whose realizations are "bad" in some technical sense, we will also have graphs, all of whose minimum-crossing drawings are also "bad" in the same sense. In particular, recent work of Goodman, Pollack, and Sturmfels implies the following remarkable result (via dualization to straight-line arrangements):

Theorem [14]. For any $n \geq 1$ there exists an arrangement $E^{n}$ of straight-line segments, such that in every straight-line realization of $E^{n}$ the coordinates of the ends require exponentially many bits.

Together with Theorem 1(ii) (with $t=1$ ), this yields:

Theorem 2. There exists an infinite family of graphs $\left\{G^{n}\right\}$ such that, in every rectilinear drawing of $G^{n}$ with $\mathrm{cr}_{1}\left(G^{n}\right)$ crossings, the coordinates of the vertices require more than polynomially many bits.

As a result, there does not exist a polynomial-time algorithm for producing a rectilinear drawing of a graph which achieves the rectilinear crossing number. Here we are assuming a model where either

(a) the coordinates of the vertices must be written down, or

(b) a physical drawing of the graph must be produced,

and drawing a graph consumes resources (such as time or space) proportional to the size of the drawing (e.g., if the graph is to be drawn on a computer screen). In a different model of graph drawing our result might not have any impact at all. We remark that the results of the paper, together with the work of Mnëv [18], imply that the problem of computing the rectilinear crossing number of a graph is as hard as solving a system of real polynomial inequalities. Furthermore, using the recent result of Shor [21], that the problem of testing whether a given arrangement of pseudolines is stretchable is NP-hard, Theorem 1-yields that testing whether for a given graph the rectilinear crossing number equals the crossing number, is also NP-hard.

Let $t \geq 1$. As shown in [1] using $t=2$ instead of $t=1$ can dramatically decrease the number of crossings (the improvement in fact cannot be bounded as a function of the size of the graph). However, for any fixed $t$ there is a result similar to Theorem 2 (given in Theorem 3). On the other hand, for any graph $G$, we can construct a 2-polygonal drawing with at most $2(\operatorname{cr}(G))^{2}$ crossings, in polynomial space (logarithmically many bits per vertex).

How complicated can a minimum-crossing drawing of a graph be? In other words, is there a fixed number $t$, so that for all graphs $G$, the crossing number of $G$ can be achieved with a $t$-polygonal drawing? We show that the answer is no, and thus, for graphs of crossing number $k$ or less, the minimum possible such $t$ depends on $k$; let us call it $t(k)$. We prove:

Theorem 4. There exist constants $c_{1}$ and $c_{2}$, so that, for every $k \geq 1$,

$$
c_{1} k^{1 / 2} \leq t(k) \leq c_{2} k^{1 / 2}
$$


To obtain the lower bound, we apply a recent result of Kratochvil and Matousek [16], and our construction in Theorem 1. The proof of the upper bound relies on a direct construction.

\section{Forcing Arrangements in Drawings of Graphs}

In this section we prove Theorem 1. For the proof we need a definition. Let $G$ be a plane graph and let $k>1$ be an integer. The $k$-thickening $G^{\prime}$ of $G$ is the plane graph obtained by replacing each edge $e=\{u, v\}$ of $G$ by a set $b(e)$ of $k$ edge-disjoint paths of length two, with ends $u$ and $v$, each homeomorphic to $e$. If $e \neq e^{\prime}$ it is assumed that the set of internal vertices of the paths in $b(e)$ is disjoint from the similar set in $b\left(e^{\prime}\right)$.

Theorem 1. Let $A$ be an arrangement of $n$ pseudolines. There exists a graph $G_{A}$ with $\mathrm{cr}\left(G_{A}\right)=5 n(n-1)$ and $\left|E\left(G_{A}\right)\right|=O\left(n^{3}\right)$, with a distinguished subset $S_{A}$ of edges, such that:

(i) In every drawing of $G_{A}$ with $\operatorname{cr}\left(G_{A}\right)$ crossings, the drawing of $S_{A}$ contains a realization of $A$. Further, $\operatorname{cr}\left(G_{A}\right)=5 n(n-1)$ and $\left|E\left(G_{A}\right)\right|=O\left(n^{3}\right)$.

(ii) Let $t \geq 1$. If $A$ has a $t$-polygonal realization, then, in every t-polygonal drawing of $G_{A}$ with $\mathrm{cr}_{i}\left(G_{A}\right)$ crossings, the drawing of $S_{A}$ contains a t-polygonal realization of $A$. Here $\mathrm{cr}_{i}\left(G_{A}\right)=5 n(n-1)$ and $\left|E\left(G_{A}\right)\right|=O\left(t n^{4}\right)\left(O\left(n^{3}\right)\right.$ for $t=1)$.

Proof. (i) The graph $G_{A}$ is obtained in several steps. See Fig. 1 for an example.

1. We begin by replacing each $x \in A$ with two copies, $x_{1}$ and $x_{2}$, drawn very close to each other. Next, we obtain a plane graph $L$ by placing a vertex at each crossing and at each end of very pseudoline. Thus, for each $x \in A, L$ contains two edge disjoint paths $p_{1}(x)$ and $p_{2}(x)$, where $p_{i}(x)$ has ends (degree one vertices) $u_{i 1}(x)$, $u_{i 2}(x)$. We assume that the labeling has been done so that, as we traverse the outer facial boundary of $L$ counterclockwise, $u_{22}(x)$ immediately follows $u_{11}(x), u_{23}(x)$ appears after $u_{22}(x)$, and $u_{12}(x)$ immediately follows $u_{23}(x)$, see Fig. 1(b)). Next, we add to $L$ a cycle $C$, joining all the vertices $u_{i k}(x)$ in the cyclic order, so as to form with $L$ a plane graph. Further, $C$ contains a vertex $v_{1}(x)$ between every two vertices $u_{11}(x), u_{22}(x)$, and a vertex $v_{2}(x)$ between every two vertices $u_{23}(x), u_{12}(x), x \in A$.

2. For each $x \in A$, we add an edge $e(x)$ with endpoints $v_{1}(x)$ and $v_{2}(x)$, and so that $e(x)$ is drawn inside $C$ and "between" $p_{1}(x)$ and $p_{2}(x)$. Let $S$ be the set of all edges $e(x), x \in A$, and $H=L \cup C \cup S$. See Fig. 1(c).

3. Take a copy $H^{\prime}=C^{\prime} \cup L^{\prime} \cup S^{\prime}$ of $H$, drawn outside $H$ (and with the obvious notation), and a matching $M$ joining the vertices of $C$ to those in $C^{\prime}$, so that $L \cup C \cup L^{\prime} \cup C^{\prime} \cup M$ is plane. We replace $C \cup C^{\prime} \cup M$, by its $m$-thickening $W$ (where $m=5 n(n-1)+1$ ) so that the edges of $W$ have no crossings at all. The resulting graph is $G_{A}$, and we set $S_{A}=S \cup S^{\prime}$. See Fig. 1(d).

Let $D_{A}$ be the drawing we have just constructed, which has $5 n(n-1)$ crossings. Suppose now that $D^{*}$ is a drawing of $G_{A}$ with $\operatorname{cr}\left(G_{A}\right) \leq 5 n(n-1)$ crossings. 

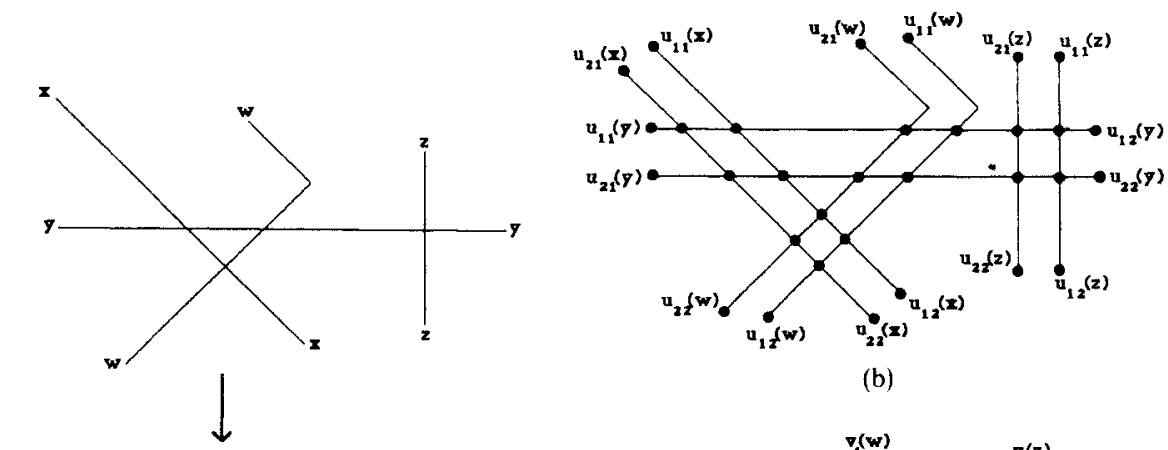

(b)

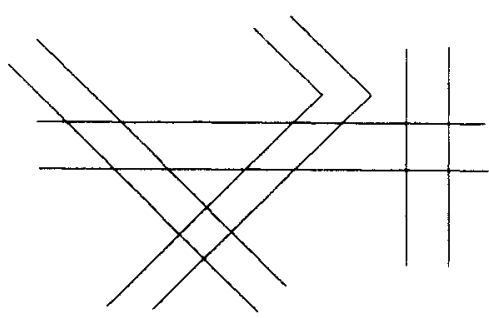

(a)

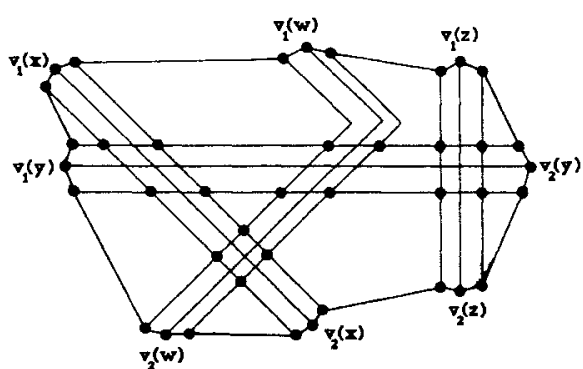

(c)

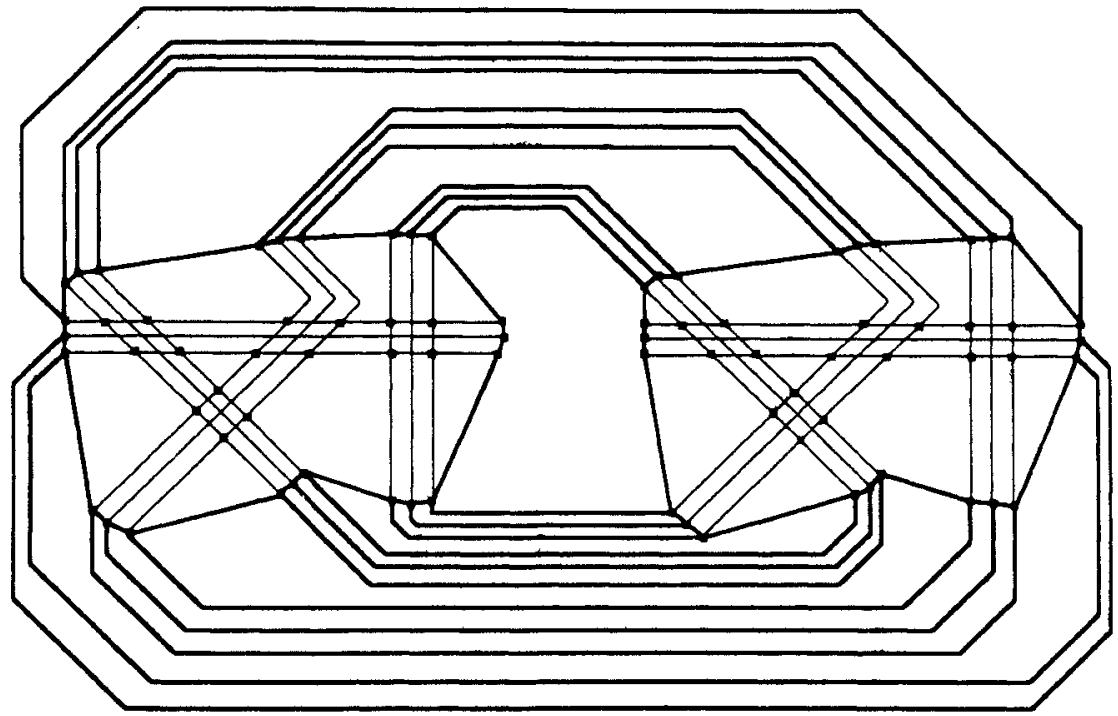

(d)

Fig. 1. (a) Doubling the arrangement. (b) Graph $L$. (c) $C \cup L \cup S$. (d) Graph $G_{A}$. Thickened edges shown as heavy lines. 
Consider an edge $t$ of $C \cup C^{\prime} \cup M$. Since $m>\operatorname{cr}\left(G_{A}\right)$, it follows that at least one of the paths in $b(t)$ has no crossings in $D^{*}$. Thus we obtain a plane drawing of $C \cup C^{\prime} \cup M$ in the obvious way. Since $C \cup C^{\prime} \cup M$ is 3-connected, it has a unique embedding, and its drawings in $D^{*}$ and $D_{A}$ are homeomorphic in the sphere. So in $D^{*}$ both $C$ and $C^{\prime}$ bound faces of $C \cup C^{\prime} \cup M$, which must contain the drawings of $C \cup L$ and $C^{\prime} \cup L^{\prime}$ (respectively). Next, let $x, y \in A$. Then, by construction, the endpoints of $e(x)$ and $e(y)$ alternate along $C$. In fact, the endpoints of $e(x)$ and the ends of each path $p_{i}(y), i=1,2$, alternate along $C$ (and similarly, the endpoints of $e(y)$ and the ends of each path $p_{i}(x), i=1,2$, alternate along $C$ ). Since, for any $z \in A, p_{1}(z)$ and $p_{2}(z)$ are edge-disjoint, we count five crossings in $D^{*}$ corresponding to the pair $x, y$ (the Jordan curve theorem). In this way we count $5 n(n-1) / 2$ crossings in the region bounded $C$. Similarly with $C^{\prime}$. Thus $5 n(n-1)=\operatorname{cr}\left(G_{A}\right)$, and the $5 n(n-1) / 2$ crossings we have just counted are all the crossings in the region bounded by $C$ (resp. $C^{\prime}$ ). Either $C$ or $C^{\prime}$ bound an inner face of $C \cup C^{\prime} \cup M$ in $D^{*}$, say $C$ does. Consequently, in $D^{*}$

(i) the drawing of $C \cup L$ is plane, and

(ii) for each $x \in A, e(x)$ does not cross any edge in $p_{i}(x), i=1,2$.

Then (ii) implies that, for each $x \in A, e(x)$ is drawn "between" $p_{1}(x)$ and $p_{2}(x)$. We conclude that the drawing of $S$ realizes $A$, as desired. This concludes the proof of (i).

(ii) Assume that the pseudolines in $A$ are $t$-polygonal. The proof of (ii) proceeds exactly as that of (i), with the exception that the initial drawing $D_{A}$ must now, in addition, be $t$-polygonal, so that we can argue that $\operatorname{cr}_{t}\left(G_{A}\right) \leq 5 n(n-1)$. It is clear that the edges of $L$ and $S$ can be assumed to be $t$-polygonal. The same holds for the edges of $C$ by drawing them "following" the outer facial cycle of $L$. Further, the edges of the matching $M$ can be drawn as $O(t n)$-polygonal lines. So let us replace each edge of $M$ by a path of length $O(t n)$, obtaining a graph $N$. The plane graph $C \cup L \cup C^{\prime} \cup L^{\prime} \cup N$, although not 3-connected, still has a unique embedding on the sphere (the only exception to 3 -connectedness are the degree-2 vertices in $N$ ). From now on we can proceed with the $m$-thickening of $C \cup C^{\prime} \cup N$, and the rest of the proof is as above. Notice that $\left|E\left(G_{A}\right)\right|=O(n m) O(t n)=O\left(t n^{4}\right)$. To obtain the reduction to $O\left(n^{3}\right)$ for the case $t=1$, notice that in this case the region enclosed by the cycle $C$ can be assumed to be a rectangle. Thus in this case the edges of $M$ need only be subdivided (at most) four times each, which yields the desired bound. This concludes the proof of (ii).

Comment. The graph $G_{A}$ has vertices of large degree, but the same result can be achieved with a graph of maximum degree 3, although the proof is somewhat longer. The construction relies on using a large section of a "wall," a special type of cubic graph.

\section{Graphs Which Require More Than Polynomially Many Bits}

Let us apply Theorem 1(ii), with $t=1$, where for $A$ we use one of the straight-line arrangements obtained from the results in [14]. We have: 
Theorem 2. There exists an infinite family of graphs $\left\{G^{n}\right\}$ such that, in every rectilinear drawing of $G^{n}$ with $\mathrm{cr}_{1}\left(G^{n}\right)$ crossings, the coordinates of the vertices require more than $\exp \left(c\left|E\left(G^{n}\right)\right|^{1 / 3}\right)$ many bits, where $c$ is a fixed constant.

Can the situation in Theorem 2 be avoided if we use $t$-polygonal drawings, where $t>1$ is "small"? The answer is no, as shown in Theorem 3 below. The proof of this theorem makes use of a certain arrangement of nine pseudolines due to Ringel [19] which is described in [15] (also see [8]). This arrangement, which we denote by $R_{9}$, does not have a straight-line realization, but on the other hand it has 2-polygonal realizations in which only one pseudoline is not 1-polygonal (i.e., all the others are straight lines. The distinguished pseudoline can be arbitrarily chosen).

Theorem 3. Let $t>1$. There exists an infinite family of graphs $\left\{G^{n, t}, n=1,2, \ldots\right\}$ such that every $t$-polygonal drawing of $G^{n, t}$ with $\mathrm{cr}_{t}\left(G^{n, t}\right)$ crossings requires more than polynomially many bits.

Proof. The proof of this (perhaps not unexpected) theorem requires an intermediate construction. Let $M$ denote an arbitrary arrangement of $n$ straight lines. For $t=1,2, \ldots$, we inductively define an arrangement $A(t)$ of $t$-polygonal pseudolines as follows. First, let $A(1)$ be a straight-line realization of $M$. Suppose now that $t>1$. Replace each pseudoline $x \in A(t-1)$ with a set $S(x)$ of nine copies, drawn very close to one another, and so that the copies of each segment of $x$ are parallel. The "spacing" between consecutive copies of $x$ is determined below.

We obtain a 2-polygonal realization of $R_{9}$ as follows. First, choose an arbitrary $y \in S(x)$, and stretch it a small distance beyond the ends of the other members of $S(x)$ (we still call the stretched pseudoline $y$ for convenience). Next, attach to the end of each pseudoline $z$ in $S(x)$ an additional (small) straight-line segment $e(z)$. Let $r(y)$ be the 2-polygonal line formed by $e(y)$ and the stretched portion of $y$. It is not hard to see that if

(1) the spacing between copies of $x$,

(2) the stretching of $y$, and

(3) the choice of segments $e(z)$

are properly carried out, then the arrangement $\{e(z): z \neq y\} \cup\{r(y)\}$ will be a 2-polygonal realization of $R_{9}$. See Fig. 2. Here $r(y)$ is the only pseudoline in this 2-polygonal realization of $R_{9}$ which is not a straight line. Notice that for each pseudoline in $S(x)$ we introduced at most one new breakpoint. Thus the pseudolines $z \cup e(z)$ are all $t$-polygonal, since $x$ is $(t-1)$-polygonal.

The arrangement which results from carrying out this operation, for every $x$, is $A(t)$, which is $t$-polygonal, as desired. The following claim is clear and we include the proof for completeness.

Claim. Every $t$-polygonal realization of $A(t)$ includes, as a subdrawing, a straightline realization of $M$. 

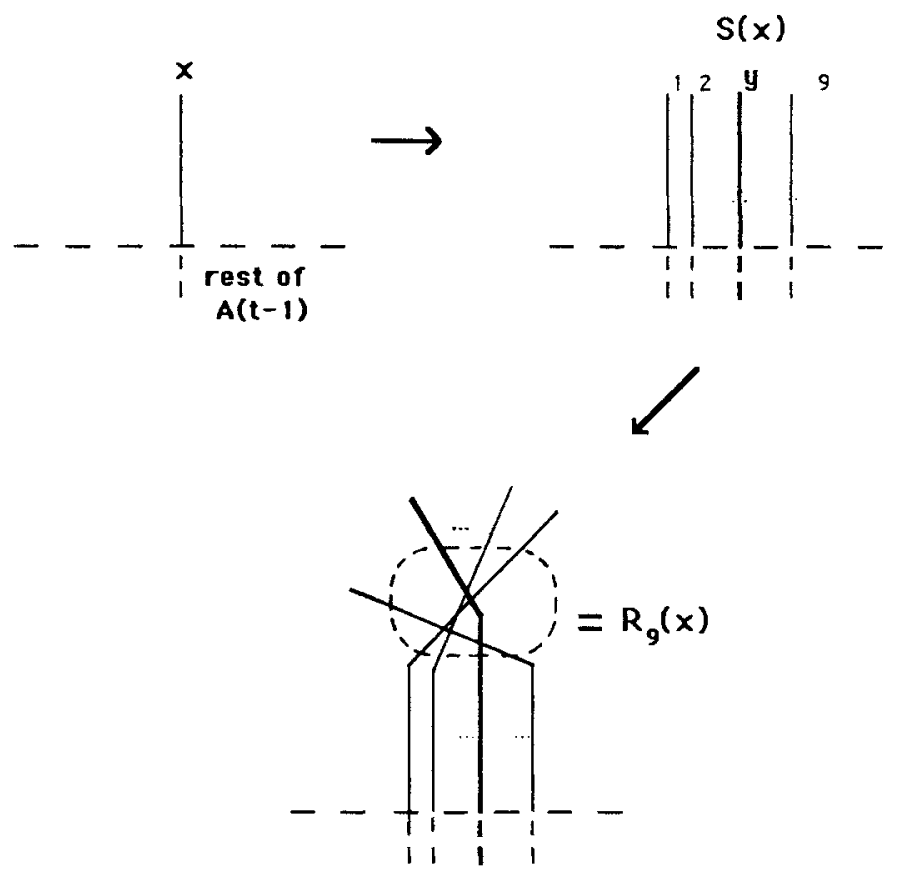

Fig. 2

Proof of the Claim. The claim is clear for $t=1$. Next, assume that $t>1$ and consider any $t$-polygonal realization $L$ of $A(t)$. Denote by $R_{9}(x)$ the portion of the pseudolines in $S(x)$ which realize $R_{9}$. Clearly, for every $x \in A(t-1)$, there is a pseudoline $z(x) \in S(x)$ which has in $L$ at least one breakpoint in the portion contained in $R_{9}(x)$ (otherwise $R_{9}(x)$ would have a straight-line realization). Thus the arrangement $\{z(x): x \in A(t-1)\}$ includes in $L$ a $(t-1)$-polygonal realization of $A(t-1)$, and the claim follows by induction. This completes the proof of the claim.

We note now that $|A(t)|=9^{t} n$. To conclude the proof of Theorem 3, let us use for $M$ the "bad" arrangement which can be constructed using the methods of [14] (i.e., an arrangement each of whose straight-line realizations requires exponentially many bits). Then we have that in every $t$-polygonal realization of $A(t)$ the coordinates of the ends and breakpoints require more than polynomially many bits. Hence, we apply the construction in Theorem 1(ii) to $A(t)$, and define $G^{n, t}=G_{A(t)}$.

The following question arises as a result of Theorems 2 and 3: what can be achieved in polynomial space? In other words, given a graph $G$ and fixed $t$, can we always provide a $t$-polygonal drawing of $G$, in polynomial space, with a number 
of crossings bounded as a function of $\operatorname{cr}_{t}(G)$ ? For $t=2$, the answer is yes, in a strong sense, as follows. In [1], the authors showed:

Theorem [1]. For every graph $G, \mathrm{cr}_{2}(G) \leq 2(\operatorname{cr}(G))^{2}$.

In fact, if $G$ has $n$ vertices, the construction in [1] yields a 2-polygonal drawing of $G$, in a grid of size $O\left(n(\operatorname{cr}(G))^{1 / 2}\right)$, with at most $2(\operatorname{cr}(G))^{2}$ crossings (we stress that this construction carries out something more difficult than what we asked for: it approximates $\operatorname{cr}(G)$ rather than $\mathrm{cr}_{2}(G)$ ). This result is based on the theorem of deFraysseix et al. [3], that any plane graph has a straight-line drawing of linear size (see also [20]). Essentially, the desired 2-polygonal drawing of $G$ is obtained from a plane drawing of a graph with $O(n)$ vertices. We refer the reader to [1] for details. Thus, for the case $t=2$, the above question has a positive answer, in a strong sense. Nevertheless, it is an open question whether the quadratic bound is best possible for $t=2$, and whether it can be improved for larger $t$. Moreover, the case $t=1$, perhaps the most interesting, is completely open. We remark that for general graphs the rectilinear crossing number is not bounded by any function of the crossing number [1], but on the other hand if we consider the class of graphs with some fixed upper bound on maximum degree, the rectilinear crossing number is at most quadratic in the crossing number [2].

As one last application of Theorem 1, we point out that the method used in the proof of Theorem 3 easily yields, for every $t>1$, a graph $H$ with $\operatorname{cr}(H)=$ $\mathrm{Cr}_{t}(H)<\mathrm{Cr}_{t-1}(H)$. By taking disjoint copies of such graphs, we obtain:

Corollary. For every $t>1$ there exists a graph $G$, with $\operatorname{cr}(G)=\operatorname{cr}_{t}(G)$, and such that, for every $t \geq i>1, \mathrm{cr}_{i}(G)<\mathrm{cr}_{i-1}(G)$.

\section{Achieving the Crossing Number with $t$-Polygonal Drawings}

In this section we study the following question: given a graph $G$, how large must $t$ be so that $\operatorname{cr}_{t}(G)=\operatorname{cr}(G)$ ? Suppose first that we are only concerned with graphs $G$ with bounded crossing number, say, at most $r$. Then, from a drawing of $G$ with $\operatorname{cr}(G)$ crossings, we obtain a drawing of a plane graph by placing a vertex at each crossing. Passing to a straight-line plane drawing of this graph, and removing the added vertices, we obtain an $r$-polygonal drawing of $G$ which attains the crossing number. But what if $\operatorname{cr}(G)$ is no longer bounded? Then, as is shown below, the smallest value $t$ so that $\operatorname{cr}_{t}(G)=\operatorname{cr}(G)$ will in general depend on $\operatorname{cr}(G)$. More formally, let

$$
\chi(k)=\{G: \operatorname{cr}(G) \leq k\}
$$

and

$$
t(k)=\min \left\{t: \operatorname{cr}_{s}(G)=\operatorname{cr}(G) \text { for all } G \in \chi(k)\right\}
$$


Then we have:

Theorem 4. There exist constants $c_{1}$ and $c_{2}$ so that, for every $k \geq 1$,

$$
c_{1} k^{1 / 2} \leq t(k) \leq c_{2} k^{1 / 2}
$$

The rest of this paper is devoted to the proof of Theorem 4 .

\subsection{The Lower Bound}

In order to obtain the lower bound, we use the following theorem, recently proved by Kratochvil and Matousek.

Theorem [16]. For each $n$, there exists an arrangement $Z(n)$ of $n$ pseudolines which cannot be realized with $t$-polygonal pseudolines unless $t \geq c n$, where $c$ is a constant.

Remark. In [16] arrangements are allowed to have pairs of pseudolines that do not meet, but this detail is easily dealt with to obtain the above theorem.

Now, given $n$, consider the graph $G_{Z(n)}$ produced by Theorem 1. Write $H=G_{Z(n)}$. We have that $\operatorname{cr}(H)=O\left(n^{2}\right)$. On the other hand, unless $t \geq c n$, we also have $\operatorname{cr}_{t}(H)>\operatorname{cr}(H)$ by definition of $Z(n)$. Thus the lower bound in Theorem 4 is proved.

\subsection{The Upper Bound}

In this section we prove the upper bound in Theorem $4, t(k) \leq O\left(k^{1 / 2}\right)$. Let $D$ be a drawing of $G$ with $\operatorname{cr}(G)=k$ crossings. We partition the edges of $G$ into (at most) two classes, $H$ and $L$, where

$$
H=\text { the set of edges with more than } 2 k^{1 / 2} \text { crossings in } D
$$

and

$$
L=\text { the set of edges with at most } 2 k^{1 / 2} \text { crossings in } D \text {. }
$$

(For convenience, we also use $H$ and $L$ to refer to the corresponding subgraphs of $G$.) Now if $H$ is empty, then we are essentially done: we obtain, from $D$, a plane graph by placing a new vertex at each crossing point, in addition to the vertices of $G$. Since any plane graph has a straight-line drawing (see [22] for a short proof), we obtain a $\left(2 k^{1 / 2}\right)$-polygonal drawing of $G$ with $\operatorname{cr}(G)$ crossings, as desired. In general, $H$ is of course nonempty, but still this basic construct is the appropriate idea to use.

Our procedure is first to draw $H$ and then $L$, always obtaining drawings homeomorphic to those in $D$. The key fact here is that $|H|<k^{1 / 2}$. So if we draw 
$H$, ignoring $L$, and use the plane graph construction as in the previous paragraph, the members of $H$ will be $\left(k^{1 / 2}+1\right)$-polygonal lines (we stress that the crossings of edges in $H$ with edges in $L$ are ignored here). Similarly, if we draw $L$, even taking into account the crossings with edges in $H$, and again use the plane graph method, the edges will be $O\left(k^{1 / 2}\right)$-polygonal lines (by definition of $L$ ). As shown below, by building more structure especially into the drawing of $H$, we are able to piece together the two drawings as desired.

Step 1: Drawing $H$. Consider the drawing of $H$ provided by $D$. In general, this drawing has several arc-connected components, which we call pieces. Each piece consists of possibly more than one graph-theoretic componet of $H$, and each edge in $H$ appears in one piece. Each edge is partitioned into sections, where a section is the portion of the edge between consecutive crossings (with other edges of $H$ ) or a portion between a vertex and the crossing closest to it. We draw each piece separately, with some added structure.

Thus, let $Z$ be a piece. Then the complement in $\mathbb{R}^{2}$ of $Z$ consists of several connected regions or faces (so if $Z$ is a plane these are faces in the standard sense). Each face is bounded by sections, although the boundary may not be simple: both sides of a section may be incident to the same face. One of the faces is unbounded, in the sense that it is homeomorphic to the complement of a closed disk. From $Z$, obtain a plane graph as follows. First, put one vertex at each crossing point (once more, these are crossings involving two edges in $H$ ), in addition to the original vertices. Thus each section becomes an edge. Next, subdivide each section $s$ by introducing one new vertex $v(s)$. Finally, for each face $F$, draw a vertex $w(F)$ in $F$. Then we join $w(F)$ to each vertex $v(s)$ (where $s$ is a bounding segment of $F$ ) with a length-two path. If the boundary of $F$ is not simple, and if both sides of a segment $s$ are incident to $F$, then we use two length-two paths from $v(s)$ to $w(F)$, drawn to each side of $s$ (see Fig. 3). All of the length-two paths are edge-disjoint, and can be drawn without crossings. We obtain a plane graph $Z^{\prime}$.

We note that the outer face of $Z^{\prime}$ contains some vertex $w(F)$. By adding edges if necessary, we may assume this outer face is a triangle. We produce a drawing of $Z$ by taking a straight-line drawing $R(Z)$ of $Z^{\prime}$. it is clear that this drawing of $z$ is $O\left(k^{1 / 2}\right)$-polygonal.

Step 2: Beginning the Drawing of $L$. In order to draw $L$, let us return to the original drawing $D$ of $G$. The complement of $H$ is partitioned into several connected regions, which we call plots. Each plot is the intersection of several faces of different pieces, as discussed above. In general, each plot $P$ will be incident to several pieces, all of whom, with at most one exception, it encloses (and the one exception encloses $P$ ). In other words, $P$ is homeomorphic to an open disk (or to $\mathbb{R}^{2}$ ) with several holes cut out (thus each boundary component of $P$ corresponds to a different piece). We draw $L$ by separately drawing the part of $L$ contained in each plot. Below we see how to piece together all of these subdrawings and also the drawing of $H$ produced above.

Thus, let $P$ be a plot and assume that $P$ contains part of the drawing of $L$ in $D$. Let us "expand" each boundary component slightly, to obtain a larger disk. Thus (for example) if $Z$ is a piece enclosed by $P$ and incident to it, then we obtain 

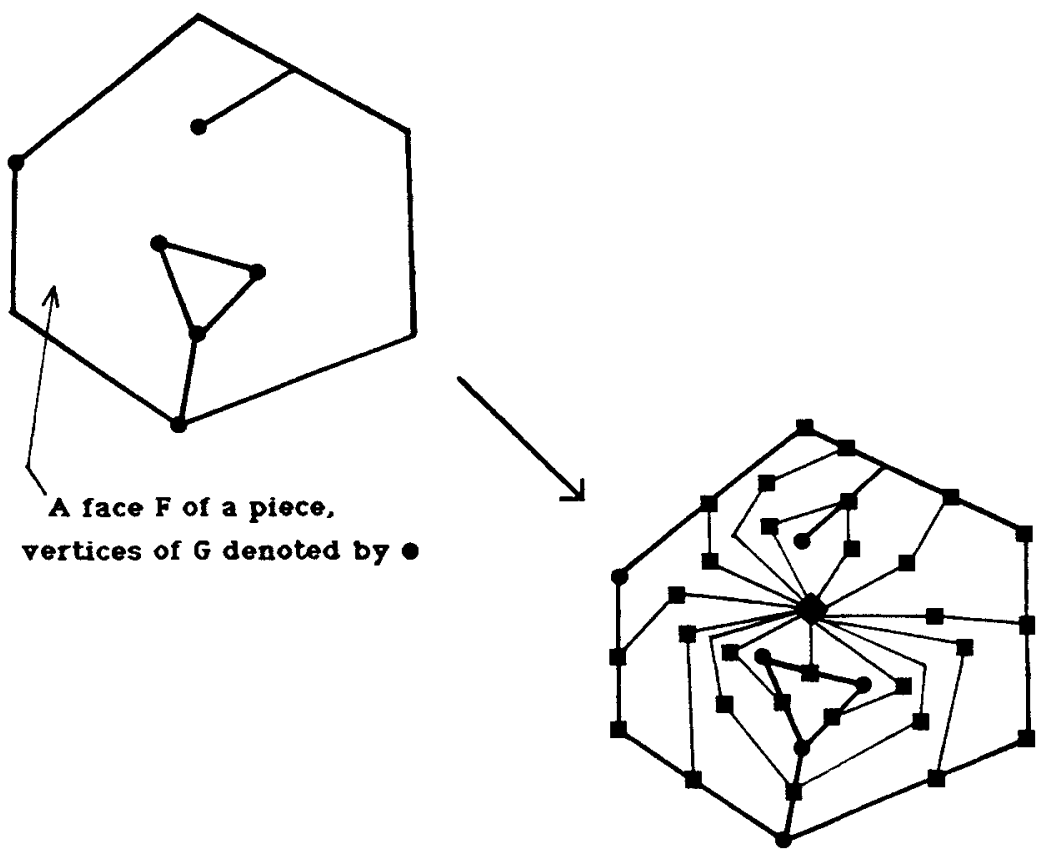

w(F) indicated by

other new vertices indicated by

Fig. 3

a disk that encloses $Z$ "very closely" (see Fig. 4(a) and (b)). We note that this expansion "truncates" portions of edges of $L$ that appear in $P$ and intersect $Z$ (we say such portions $\operatorname{link} Z$ and $P$ ). Let $P^{\prime}$ be the subset of $P$ resulting from the removal of the expanded boundary components. We assume that the expansions are small enough, so that all vertices and crossing points contained in $P$ are also contained in $P^{\prime}$.

Consider the subdrawing of $L$ contained in $P^{\prime}$. From this subdrawing obtain a plane graph by placing a vertex at each crossing point, in addition to any vertices of $G$. Further, if $Z$ is a piece, the subset in $P^{\prime}$ of each portion that links $Z$ and $P$, terminates at some point along the expanded boundary of $Z$. At each such termination point, we place one new vertex, called a termination vertex (so, at this stage, each termination vertex has degree one). Finally, we add an additional vertex $v(Z)$ and edges joining $v(Z)$ to all termination vertices corresponding to $Z$ (so now each termination vertex has degree two). As noted above, there is at most one piece $Z$ which encloses $P$. If such is the case, the plane graph we have constructed so far has the vertex $v(Z)$ in its outer face. We triangulate this face, making sure that $v(Z)$ remains in the outer face. This concludes the construction of the plane graph corresponding to $P$ (see Fig. 4(c)). Let $S(P)$ denote a straight-line drawing of this plane graph (for convenience, we use $S(P)$ to refer to the graph itself). 


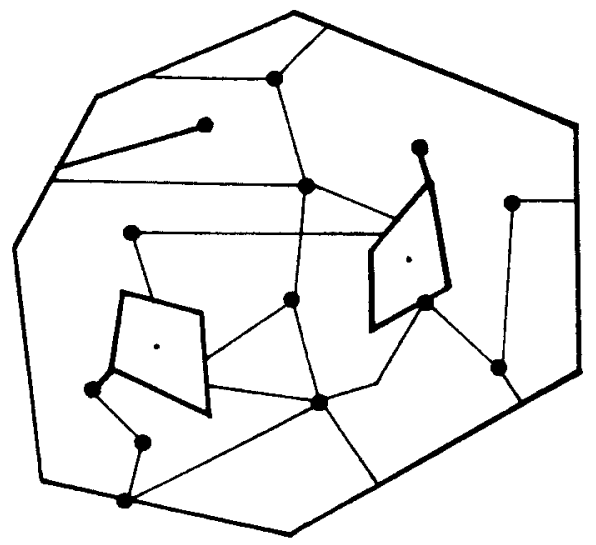

(a)

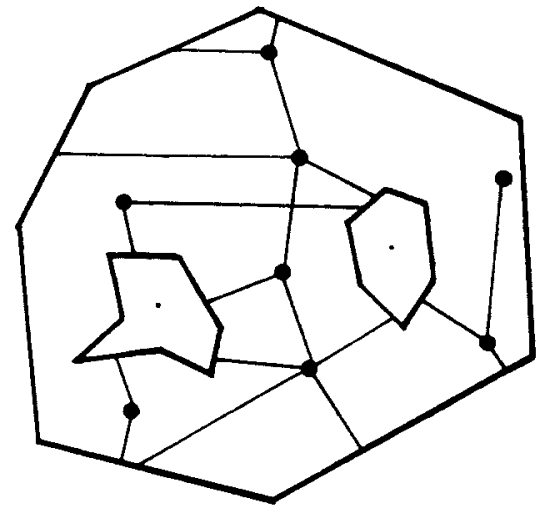

(b)

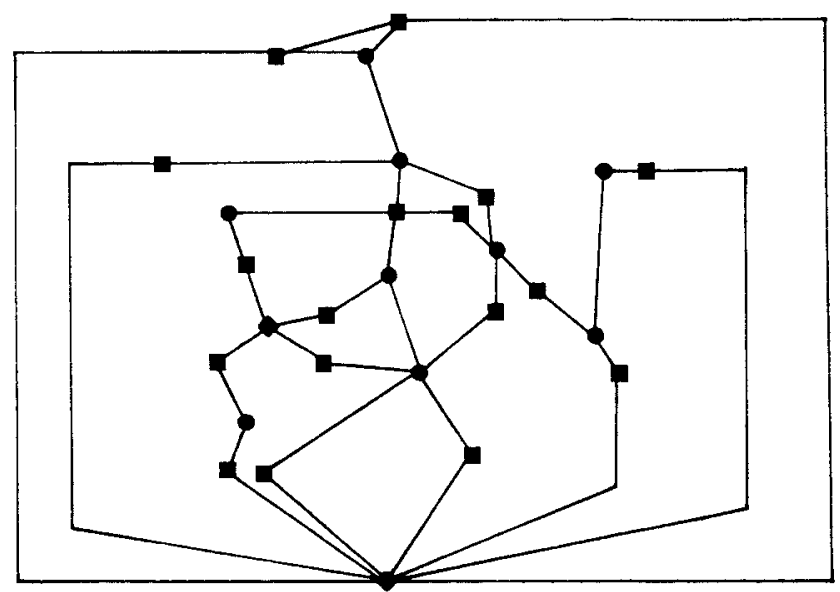

(c)

Fig. 4. (a) A plot that encloses two pieces and is enclosed by one. (b) After expanding the boundaries. (c) The resulting plane graph. $\bullet$, vertices of type $v(z)$; $\mathbf{a}$, other added vertices.

We remark that, for every piece $Z$ incident to $P$, the linking portions of $Z$ and $P$ intersect the boundary of $Z$ with a certain clockwise ordering. This is isomorphic to the clockwise ordering of the corresponding edges incident to $v(Z)$.

Step 3: Putting $L$ and $H$ Together. The notion of enclosure can be used to define a partial order on the plots. $(A>B$ if $A$ encloses $B$. It is not difficult, but tedious, actually to prove this is a partial order.) We put together the various drawings $S(P)$ and $R(Z)$ by moving downward in the parital order.

Thus, let $P$ be a plot maximal in the partial order. Start with the graph $S(P)$. Consider first a piece $Z$ enclosed by $P$ and incident to it. Let us draw a very small triangle around $v(z)$ and remove its interior (so the edges incident to $v(z)$ in $S(P)$ 
are truncated). Inside the resulting triangle we place a small copy of the drawing $R(Z)$. We recall that $R(Z)$ contains a certain vertex $w(F)$, contained in the outer face of $F$, which is connected (via length-two paths) to the vertices $v(s)$, for all boundary sections $s$ of $Z$. Further, the outer face of $R(Z)$ is a triangle.

Next we draw the portions that link $P$ and $Z$. Consider any given linking portion $i$ that (say) terminates on some boundary segment $s$ of $Z$. Now, corresponding to $i$, there is some edge $e_{i}$ incident to $v(Z)$ which is truncated by the small triangle around $v(Z)$. The idea is that we now extend the edge $e_{i}$ into the triangle, to a point arbitrarily close to $w(F)$, and then along the appropriate length-two path in $R(Z)$ to the section $s$ (See Fig. $5(\mathrm{a})-(\mathrm{d})$ ).

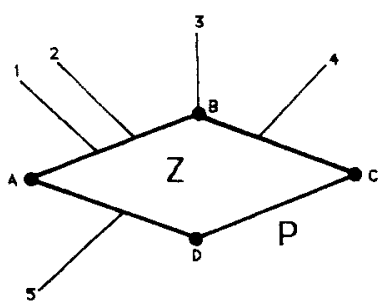

(a)

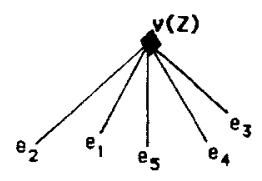

(b)

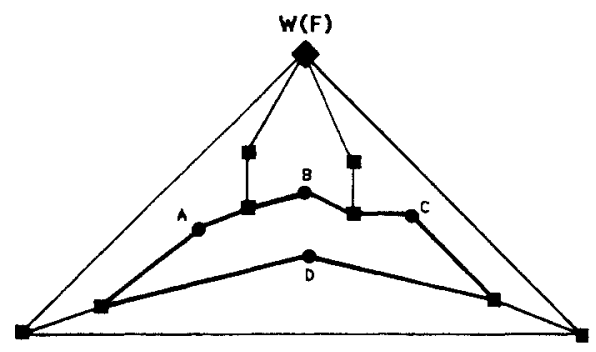

(c)

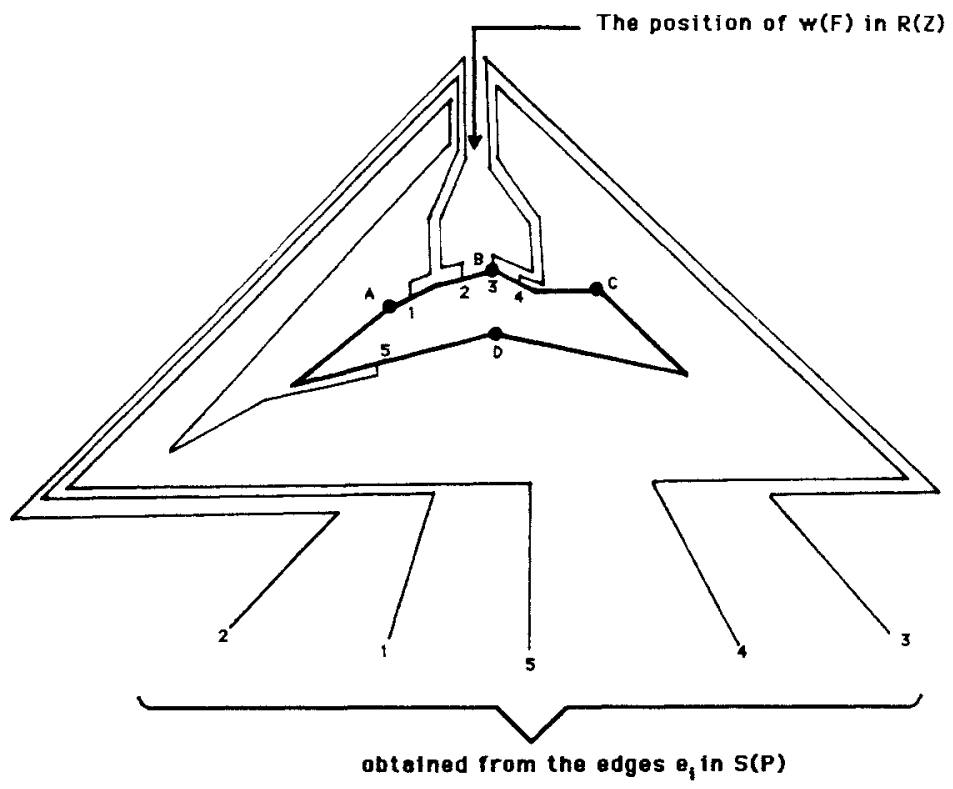

(d)

Fig. 5. (a) Original drawing of $Z$ and $P$ showing only the boundary sections of $Z$ (heavy lines) and only the linking sections of $Z$ and $P$ (light lines). (b) Part of drawing $S(P)$. (c) Part of drawing $R(Z)$. (d) Integrating the drawings of $Z$ and $P$. 
Can we simultaneously carry this out for all linking sections $i$ of $P$ and $Z$ without introducing new crossings? The answer is yes, and there are two facts that ensure this:

(1) The clockwise ordering of the linking sections along the boundary of $Z$ is isomorphic to the clockwise ordering of the edges incident to $v(z)$, in $S(P)$.

(2) $P^{\prime}$ contains all crossing points of edges of $L$ that were contained in $P$.

Thus, in fact, the simultaneous drawing can be carried out, which yields a drawing of all the linking sections that are homeomorphic to that in $D$.

It is clear that the drawings of the linking portions can be done with polygonal lines. But how many breakpoints are involved? First, to go from the boundary of the triangle enclosing $v(Z)$ to the vicinity of $w(F)$, we only need $O(1)$ breakpoints (we wrap around the triangle until we get to a point that sees $w(F)$, and then we extend from that point to $w(F)$ ). Further, we can go from $w(F)$ to each boundary section $s$ with at most $O(1)$ additional breakpoints. Thus we overall need $O(1)$ break points per linking portion. Notice that each boundary section $s$ will in general contain points of several linking portions, in some permutation.

This concludes the description of how to put together the drawings $R(Z)$ and $S(P)$, in the case where $P$ encloses $Z$. The reverse case is simpler and similar, and is left to the reader (here we would take a small copy of $S(P)$ and place it "at" the corresponding vertex $w(F)$ ).

Having handled $P$, we then recursively handle the other plots, by moving down the partial order given by enclosure. The "freedom" mentioned at the end of the penultimate paragraph guarantees that the recursive process can indeed be carried out, yielding at the end a polygonal drawing of $G$ with $\operatorname{cr}(G)$ crossings.

Counting Breakpoints. We saw in Step 1 that every edge of $H$ is drawn as an $O\left(k^{1 / 2}\right)$-polygonal line. How about the edges of $L$ ? These edges have two types of breakpoints:

(1) Those produced when drawing the graphs $S(P)$.

(2) Those produced when drawing the linking sections.

Consider an edge $e$ of $L$. Then the total number of type (1) breakpoints on this edge is at most of the order of the number of crossings on this edge (recall how $S(P)$ was constructed), which is $O\left(k^{1 / 2}\right)$ by definition of $L$. Each linking section contained in $e$ also contributes $O(1)$ breakpoints. But for each linking section we also count an additional crossing on $e$ (or an endpoint of $e$ ). Thus again we count at most $O\left(k^{1 / 2}\right)$ additional breakpoints. Hence every edge is drawn as an $O\left(k^{1 / 2}\right)$ polygonal line, as desired.

This concludes the proof of the upper bound $t(k) \leq O\left(k^{1 / 2}\right)$.

\section{Future Problems}

There are several open problems that are worth mentioning here. One has already been mentioned above; namely, the approximation of $t$-polygonal crossing numbers in polynomial space, or the aproximation of the crossing number using 
$t$-polygonal drawings with $t$ small and in polynomial space. Another intriguing possibility is the use of systems of polynomials (similar to those used in the lower bound in Theorem 4) to drive polynomial-time heuristics for an approximation of the crossing numbers. Such heuristics would relay on approximate algorithms for solving systems of polynomial inequalities; a problem that has itself been recognized as very difficult by various researchers. Yet another possible tool to approximate the crossing number would be to use, instead of $t$-polygonal lines, polynomial lines (graphs of polynomials in one variable) of small degree. See [11] for results on realizations of arrangements of pseudolines with polynomial lines. From the point of view of wiring applications, a perhaps more useful approach would be to use splines of polynomials.

\section{Acknowledgments}

I would like to thank N. Alon, J. E. Goodman, and R. Pollack for useful discussions, and Peter Shor and the referee for pointing out Mnëv's paper.

\section{References}

1. D. Bienstock and N. Dean, Bounds on rectilinear crossing numbers, submitted.

2. D. Bienstock and N. Dean, New results on polygonal crossing numbers, submitted.

3. H. deFraysseix, R. Pollack, and J. Pach, Small sets supporting Fáry embeddings of planar graphs, Proc. 20th STOC (1988), pp. 426-433.

4. P. Erdös and R. K. Guy, Crossing number problems, Amer. Math. Monthly 80 (1973), 52-58.

5. M. R. Garey and D. S. Johnson, Crossing number is NP-complete, SIAM J. Algebraic Discete Methods 4 (1983), 312-316.

6. J. E. Goodman, Proof of a conjecture of Burr, Grünbaum, and Sloane, Discrete Math. 32 (1980), $27-35$.

7. J. E. Goodman and R. Pollack, On the combinatorial classification of nondegenerate configurations in the plane, J. Combin. Theory Ser. $A 29$ (1980), 230-235.

8. J. E. Goodman and R. Pollack, Proof of Grünbaum's conjecture on the stretchability of certain arrangements of pseudolines, J. Combin. Theory Ser. A 29 (1980), 385-390.

9. J. E. Goodman and R. Pollack, Multidimensional sorting, SIAM J. Comput. 12 (1983), 484-501.

10. J. E. Goodman and R. Pollack, Semispaces of configurations, cell complexes of arrangements, $J$. Combin. Theory Ser. A 37 (1984), 257-293.

11. J. E. Goodman and R. Pollack, Polynomial realization of pseudoline arrangements, Comm. Pure Appl. Math. XXXVIII (1985), 725-732.

12. J. E. Goodman and R. Pollack, There are asymptotically fewer polytopes than we thought, Bull. Amer. Math. Soc. 14 (1986), 127-129.

13. J. E. Goodman and R. Pollack, Upper bounds for configurations and polytopes in $\mathbb{R}^{d}$, Discrete Comput. Geom. 1 (1986), 229-237.

14. J. E. Goodman, R. Pollack, and B. Sturmfels, Coordinate representation of order types requires exponential storage, Proc. 22st STOC (1989), pp. 405-410.

15. B. Grünbaum, Arrangements and Spreads, CBMS Regional Conference Series in Applied Mathematics, Vol. 10, American Mathematical Society, Providence, RI (1972).

16. J. Kratochvil and J. Matousek, Intersection graphs of segments, submitted.

17. F. T. Leighton, Complexity Issues in VLSI, MIT Press, Cambridge, MA (1983). 
18. N. E. Mnèv, The universality theorems on the classification problem of configuration varieties and convex polytopes varieties, in Topology and Geometry-Rohlin Seminar (O. Ya. Viro, ed.), Lecture Notes in Mathematics, Vol. 1346, Springer-Verlag, Berlin (1988), pp. 527-544.

19. G. Ringel, Teilungen der Ebene durch Geraden oder topologische Geraden, Math. Z. 64 (1956), $79-102$.

20. W. Schnyder, Embedding planar graphs on the grid, Preliminary report, Louisiana State University, Baton Rouge.

21. P. Shor, Stretchability of pseudolines is NP-Hard, Manuscript (1990).

22. C. Thomassen, Rectilinear drawings of graphs, J. Graph Theory 12 (1988), 335--341.

23. W. T. Tutte, Toward a theory of crossing numbers, J. Combin. Theory 8 (1970), 45-53.

Received December 19, 1989, and in revised form February 28, 1990, and April 22, 1990. 